\section{Alopecia por diluição da cor: relato de caso}

\author{
Color dilution alopecia: case report
}

\author{
Natália Lôres Lopes*, André de Paula Peixoto², Thiago Souza Costa', Rafaela Bruno Faria Pisani', \\ Carolina de Moraes Barbalho Trivisol Medeiros² ${ }^{2}$ Regina Ruckert Ramadinha ${ }^{3}$, Mariana Bezerra Mascarenhas ${ }^{4}$ \& \\ Julio Israel Fernandes ${ }^{3}$ \\ 'Médicos veterinários, MSc. Programa de Pós-graduação em Medicina Veterinária - PPGMV, Universidade Federal Rural do Rio \\ de Janeiro - UFRRJ, Seropédica, RJ, Brasil \\ ${ }^{2}$ Médicos veterinários, Programa de Residência Média em Área Profissional da Saúde, Universidade Federal Rural do Rio de \\ Janeiro - UFRRJ, Seropédica, RJ, Brasil \\ ${ }^{3}$ Médicos veterinários, DSc. Departamento de Medicina e Cirurgia Veterinária, Universidade Federal Rural do Rio de Janeiro - \\ UFRRJ, Seropédica, RJ, Brasil \\ 4Médica veterinária, DSc. Programa de Pós-graduação em Medicina Veterinária - PPGMV, Universidade Federal Rural do Rio de \\ Janeiro - UFRRJ, Seropédica, RJ, Brasil
}

\section{Resumo}

A alopecia por diluição da cor é caracterizada por displasia folicular que leva à alopecia caracterizada pela distribuição anormal de melanina no pelo. Há poucos relatos em cães sem raça definida e o diagnóstico pode ser obtido baseado no tricograma e confirmado através de avaliação histopatológica. O objetivo deste estudo foi relatar um caso de alopecia por diluição da cor em um cão sem raça definida atendido no Hospital Veterinário da Universidade Federal Rural do Rio de Janeiro que apresentava hipotricose generalizada. Foi realizado tricograma e observou-se a presença de agregados de melanina ao longo das hastes pilosas. O exame histopatológico apresentou macromelanossomas atípicos e plugues de melanina em estruturas foliculares espalhadas por toda a derme superficial e profunda, ligeira acantose com hiperqueratose e ausência de muitos folículos. As alterações observadas foram consistentes com alopecia por diluição da cor. Conclui-se que para se obter o diagnóstico da alopecia por diluição da cor é importante o histórico, um exame clínico e dermatológico bem feito, com a exclusão de possíveis diagnósticos diferencias além da histopatologia de pele que é uma importante ferramenta para auxiliar no diagnóstico.

Palavras-chave: cão, alopecia, displasia follicular.

\section{Abstract}

Color dilution alopecia is characterized by follicular dysplasia that leads to alopecia characterized by an abnormal distribution of melanin in the hair. There are few reports of mixed breed dogs and the diagnosis can be reached based on trichogramma and confirmed by histopathological evaluation. The aim of this study was to report a case of Color dilution alopecia in a mixed breed dog attended at the Veterinary Hospital of the Federal Rural University of Rio de Janeiro that had generalized hypotrichosis. Trichogramma was performed and it was observed the presence of melanin aggregates along the hair shafts by the tricogram analysis. Histopathological evaluation showed the presence of atypical macromelanossomas and melanin plugs into follicular structures scattered throughout the superficial dermis and deep, mild acanthosis with hyperkeratosis and absence of many follicles. The changes observed were consistent with color diluted alopecia. It is concluded that in order to obtain the diagnosis of color diluted alopecia, clinical history is important as well as a well-done clinical and dermatological examination, with the exclusion of possible diagnoses and the skin histopathology, which is an important tool for diagnosis.

Keywords: dog, alopecia, follicular dysplasia.

\section{Introdução}

A alopecia por diluição da cor é uma dermatopatia hereditária relativamente incomum, caracterizada por displasia folicular (Gross et al., 2005) associada a distribuição anormal de melanina nos pelos (Medleau \& Hnilica, 2003). Ocorre em cães com pelagem azul ou avermelhada (Miller et al., 2013). É mais relatada em doberman pinschers (Ferreira et al., 2007; Kim et al., 2005; Madewell et al., 1997; Miller et al., 2013), mas também já foi descrita em teckel (Beco et al., 1996; Bombassaro, 2012; Kim et al., 2005), schnauzer (Kim et al., 2005), yorkshire (Clerot \& Oliveira, \section{BJ M

Como citar: Lopes, N. L., Peixoto, A. P., Costa, T. S., Pisani, R. B. F., Medeiros, C. M. B. T., Ramadinha, R. R., Mascarenhas, M. B., \& Fernandes, J. I. (2017) Alopecia por diluição da cor: relato de caso. Brazilian Journal of Veterinary Medicine, 39(3), 198-202. doi: 10.29374/2527-2179.bjvm014117

Fonte de financiamento: Hospital Veterinário, UFRRJ.

Conflito de interesses: Os autores declaram não haver conflito de interesses que precisam ser informados.

Recebido: Maio 12, 2017.

Aceito: Junho 26, 2017.

O estudo foi realizado na Universidade Federal Rural do Rio de Janeiro - UFRRJ, Seropédica, RJ, Brasil.

\section{${ }^{*}$ Correspondência}

Natália Lôres Lopes

Hospital Veterinário de Pequenos Animais,

Universidade Federal Rural do Rio de Janeiro - UFRRJ

Rod. BR 465, km 07

CEP 23890-000 - Seropédica (RJ), Brasil

E-mail: natloresvet@gmail.com 
2004; Roperto et al., 1995) e há poucos relatos em cães mestiços (Finnie \& Tham, 1993; Miller, 1991; Perego et al., 2009).

Com etiologia ainda não muito esclarecida (Mecklenburg, 2006), acredita-se que seja decorrente de um problema primitivo no folículo piloso de origem genética (Ferreira et al., 2007), onde genes da coloração do pelo desempenham um papel significativo na condição (Miller et al., 2013). Estudos evidenciam que mutações no gene MLPH são responsáveis pela diluição da cor (Drögemüller et al., 2007; Philipp et al., 2005).

A perda de pelo geralmente começa na linha média dorsal e, muitas vezes poupa a cabeça, cauda e membros. A alopecia pode ser explicada pela fratura das hastes pilosas e por exibir folículos pilosos que não estão em crescimento (Mecklenburg, 2006). Pode ocorrer foliculite bacteriana, hipotricose (Miller et al., 2013) e há descrição de alopecia parcial e disqueratinização (Ferreira et al., 2007).

O diagnóstico baseia-se no histórico, sinais clínicos, exclusão dos diagnósticos diferenciais (Medleau \& Hnilica, 2003), na análise microscópica dos pelos, eno exame histopatológico (Gross et al., 2005; Miller et al., 2013). A microscopia dos pelos que pode ser feita através do tricograma revela a presença de macromelanossomas de formas irregulares e tamanhos distribuídos desigualmente ao longo da haste (Miller et al., 2013),

Não se conhece tratamento específico que reverta ou previna a perda de pelos (Medleau \& Hnilica, 2003), sendo limitado ao controle da descamação, do prurido (Kim et al., 2005) e da foliculite bacteriana quando ocorre (Miller et al., 2013). O uso da melatonina foi descrito, porém não demonstrou melhora clínica (Perego et al., 2009).

O objetivo deste trabalho é relatar um caso de alopecia por diluição da cor em cão Sem Raça Definida.

\section{Histórico}

Um cão sem raça definida (SRD), macho, castrado, com aproximadamente três anos de idade e de pelagem cinza foi atendido no Hospital Veterinário da Universidade Federal Rural do Rio de Janeiro. Segundo a tutora, o animal foi encontrado na rua há cinco meses com queda de pelos e ausência de prurido e não sabia mais sobre o histórico do mesmo. No exame físico foi observada pelagem ressecada, hipotricose generalizada, mais acentuada no dorso, além de descamação furfurácea (Figura 1). Não foi observada a presença de ectoparasitas.

Foi realizado exame parasitológico da raspado cutâneo que foi negativo para ácaros, cultura fúngica para dermatófitos também negativa e dosagem de T4 livre que estava dentro dos valores de referência para cães (1,12 ng/dL). Na realização do tricograma foi observada a presença de vários agregados de melanina ao longo da haste pilosa (Figura 2). Foi então realizada biópsia de pele para análise histopatológica que apresentou formação de macromelanossomas atípicos e plugs de melanina dentro de estruturas foliculares e dispersos pela derme superficial e profunda, leve acantose com hiperqueratose e ausência de pelos em muitos folículos como alterações. O diagnóstico foi compatível com alopecia por diluição da cor (Figura 3).

Após o diagnóstico foi instituído tratamento com reposição com ômega 3 diário, durante 30 dias banhos semanais com xampu contendo ureia e glicerina, além do uso de hidratante a base de óleo de macadâmia, ceramida e silicones após os banhos. Dois meses após início do tratamento, na revisão, o animal continuava a apresentar áreas de hipotricose bilateral simétrica, porém foi observada uma redução considerável da descamação apresentada no primeiro dia de atendimento (Figura 4).

\section{Discussão}

Baseando-se nos sinais clínicos, na coloração da pelagem do cão e nos exames complementares o animal foi diagnosticado com alopecia por diluição da cor. 


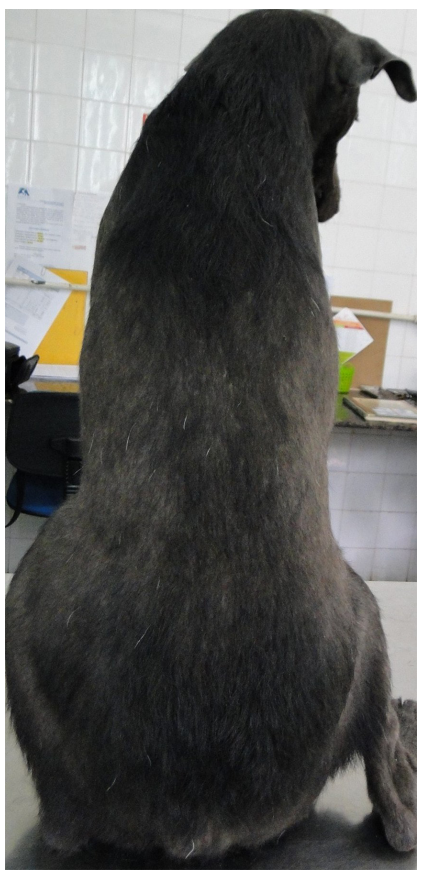

Figura 1. Canino SRD, diagnosticado com alopecia por diluição da cor, atendido no setor de Dermatologia do Hospital Veterinário da Universidade Federal Rural do Rio de Janeiro apresentando areas de hipotricose.

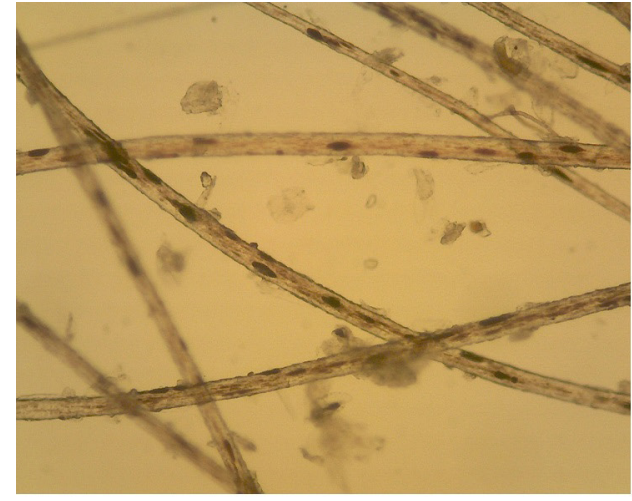

(a)

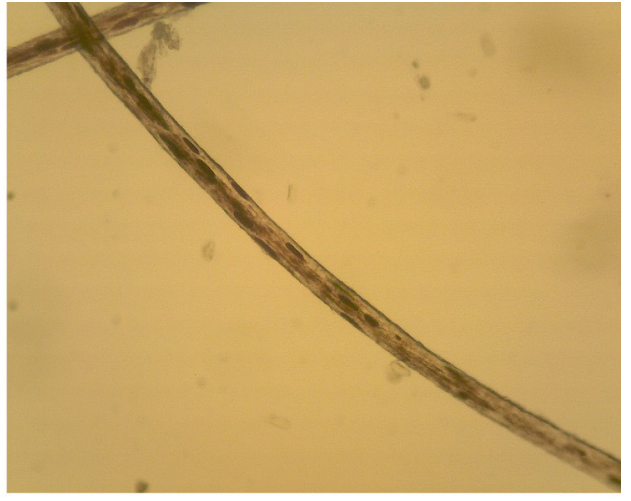

(b)

Figura 2. Agregados de Melanina observados no tricograma realizado em cão SRD diagnosticado com Alopecia por Diluição da cor.

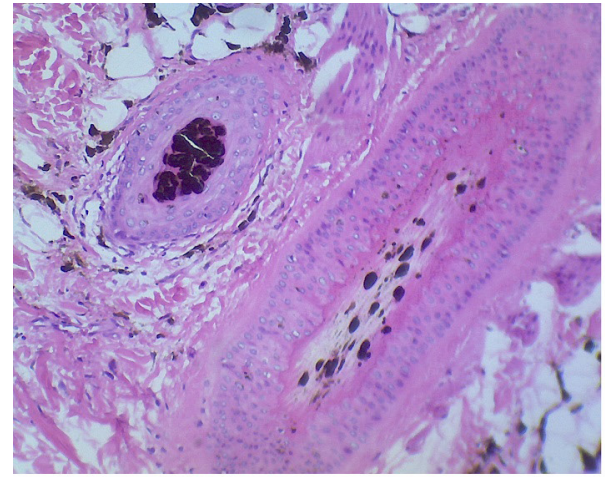

(a)

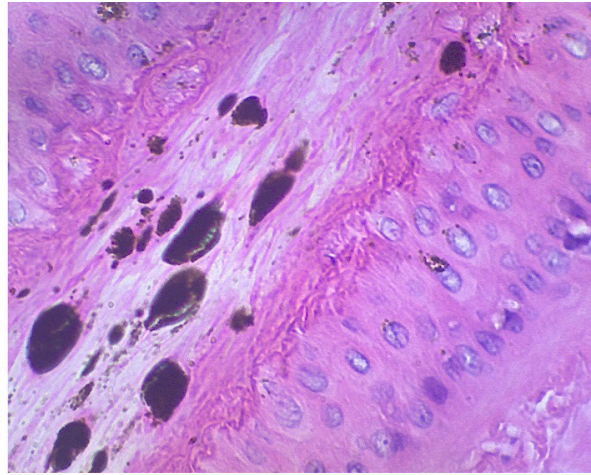

(b)

Figura 3. Exame histopatológico de cão SRD diagnosticado com Alopecia por Diluição da cor demonstrando agregados de melanina no folículo piloso. 


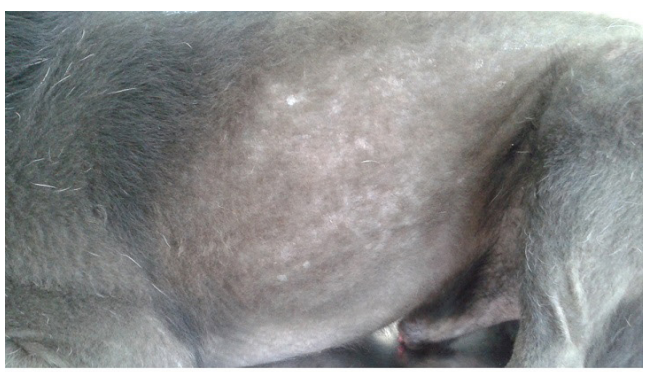

(a)

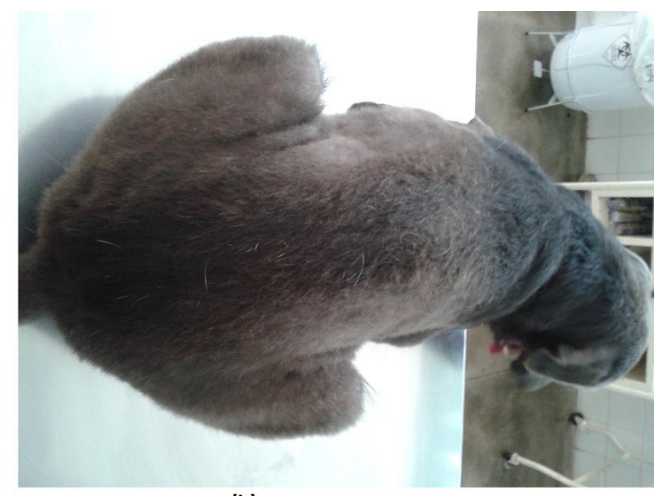

(b)

Figura 4. Canino SRD diagnosticado com Alopecia por Diluição da Cor apresentando areas de hipotricose após dois meses de tratamento na revisão.

O cão apresentava hipotricose e alopecia nas áreas de pelagem diluída assim como descrito em outros relatos desta dermatopatia (Bombassaro, 2012; Ferreira et al., 2007). A alopecia nestes casos está ligada a fratura da haste pilosa (Mecklenburg, 2006; Miller et al., 2013) e com o tempo a tendência do pelo em crescer novamente diminui (Miller et al., 2013). Os pelos de coloração normal não são atingidos (Medleau \& Hnilica, 2003). Em alguns casos os animais apresentam infecção bacteriana (Bombassaro, 2012; Perego et al., 2009), diferentemente do caso relatado, no qual o animal não apresentava sinais de piodermite.

Outras causas de alopecia e hipotricose foram descartadas através de exames complementares como raspado cutâneo e cultura para fungos, além de teste hormonal para descartar o hipotireoidismo.

Em animais entre dois a três anos ou mais as desordens endócrinas, especialmente o hipotireoidismo, devem ser consideradas (Miller et al., 2013). No presente relato esta endocrinopatia foi descartada através da dosagem de T4 livre, que se apresentou dentro dos limites de referência.

O tricograma pode ajudar a diagnosticar a alopecia por diluição da cor (Mueller, 2000). A presença de macromelanossomas observados na avaliação microscópica da haste pilosa, tricograma, foi consistente com os já relatados pela literatura, no qual observou-se a distribuição de numerosos macromelanossomas pela haste pilosa (Ferreira et al., 2007; Miller et al., 2013; Perego et al., 2009).

Assim como observado por Kim et al. (2005) a histopatologia não foi diagnóstica por si só, mas foi muito útil para estabelecer o diagnóstico definitivo. Os achados obtidos na histopatologia estavam de acordo com os descritos pela literatura nos casos de alopecia por diluição da cor (Gross et al., 2005; Miller et al., 2013). Agregados de melanina característicos na epiderme indicam que a coloração diluída está presente (Gross et al., 2005). A ausência de pelos em muitos folículos, também observada no exame histopatológico, corrobora com o descrito por Gross et al. (2005), que relataram que as hastes pilosas estão muitas vezes ausentes ou marcadamente atenuadas.

Algumas das características da alopecia por diluição da cor não são de significado patológico, mas indicam cor de pelagem diluída, especificamente aglomeração de melanina na epiderme basilar, bulbo piloso e haste pilosa (Gross et al., 2005), por isso é importante basear-se no histórico, sintomas e fazer a exclusão de outros diferenciais para realizar o diagnóstico (Medleau \& Hnilica, 2003).

Como há relato de que o uso da melatonina não trouxe melhoras para o crescimento dos pelos (Perego et al., 2009) optou-se apenas pelo tratamento sintomático com o uso de xampu para o controle da descamação e hidratação da pele. A terapia tópica foi importante para a redução da descamação observada, porém como esperado, não ocorreu a repilação das áreas de hipotricose.

\section{Conclusão}

Com base no relatado conclui-se que para se obter o diagnóstico da alopecia por diluição da cor é importante o histórico, um exame clínico e dermatológico bem feito, com a exclusão de possíveis diagnósticos diferencias além da histopatologia de pele que é uma importante ferramenta para auxiliar no diagnóstico. 


\section{Referências}

Beco, L., Fontaine, J., Gross, T. L., \& Charlier, G. (1996). Colour dilution alopecia in seven Dachshunds. A clinical study and the hereditary, microscopical and ultrastructural aspect of the disease. Veterinary Dermatology, 7(2), 91-97. http://dx.doi.org/10.1111/j.1365-3164.1996.tb00232.x.

Bombassaro, M. S. (2012). Alopecia por diluição de cor: um relato de caso (Trabalho de conclusão de curso). Universidade Castelo Branco, Campo Grande.

Clerot, L. L., \& Oliveira, R. (2004). Alopecia por diluição da cor em fêmea yorkshire terrier: relato de caso. Arquivos de Ciências Veterinárias e Zoologia, 7(2), 23.

Drögemüller, C., Philipp, U., Haase, B., Günzel-apel, A. R., \& Leeb, T. (2007). A noncoding melanophilin gene (MLPH) SNP at the splice donor of exon 1 represents a candidate causal mutation for coat color dilution in dogs. The Journal of Heredity, 98(5), 468-473. http://dx.doi.org/10.1093/jhered/esm021. PMid:17519392.

Ferreira, R. R., Machado, M. L. S., Aguiar, J., Spananberg, A., Bianchi, S. P., Oliveira, E. C., \& Driemeier, D. (2007). Displasias foliculares ligadas a cor da pelagem em cães: displasia folicular dos pelos pretos e alopecia por diluição da cor. Acta Scientiae Veterinariae, 35(1), 119-124.

Finnie, J. W., \& Tham, V. L. (1993). Colour mutant alopecia in a Kelpie × Border Collie dog. Australian Veterinary Journal, 7O(10), 388-389. http://dx.doi.org/10.1111/j.1751-0813.1993.tb00822.x. PMid:8257319.

Gross, T. L., Ihrke, P. J., Walder, E. J., \& Affolter, V. K. (2005). Skin diseases of the dog and cat: clinical and histopathologic diagnosis (2. ed.). Ames: Blackwell Science. http://dx.doi.org/10.1002/9780470752487.

Kim, J. H., Kang, K. I., Sohn, H. J., Woo, G. H., Jean, Y. H., \& Hwang, E. K. (2005). Color-dilution alopecia in dogs. Journal of Veterinary Science, 6(3), 259-261. PMid:16131833.

Madewell, B. R., Ihrke, P. J., \& Griffey, S. M. (1997). Multiple skin tumours in a Doberman Pinscher with colour dilution alopecia. Veterinary Dermatology, 8(1), 5942. http://dx.doi.org/10.1111/j.1365-3164.1997.tb00265.x.

Mecklenburg, L. (2006). An overview on congenital alopecia in domestic animals. Veterinary Dermatology, 17(6), 393-410. http://dx.doi.org/10.1111/j.1365-3164.2006.00544.x. PMid:17083571.

Medleau, L., \& Hnilica, K. A. (2003). Dermatologia de pequenos animais atlas colorido e guia terapêutico (p. 353). São Paulo: Roca.

Miller, W. H. (1991). Alopecia associated with coat colour dilution in two Yorshire Terriers, one Saluki and one mix-breed. Journal of the American Animal Hospital Association, 27, 39-43.

Miller, W. H., Griffin, C. E., \& Campbell, K. L. (2013). Muller and Kirk's small animal dermatology (7. ed., p. 948). St. Louis: Elsevier.

Mueller, R. S. (2000). Dermatology for the small animal practitioner (p. 150). Wyoming: Teton NewMedia.

Perego, R., Proverbio, D., Roccabianca, P., \& Spada, E. (2009). Color dilution alopecia in a blue Doberman pinscher crossbreed. The Canadian Veterinary Journal, 50(5), 511-514. PMid:19436637.

Philipp, U., Hamann, H., Mecklenburg, L., Nishino, S., Mignot, E., Günzel-apel, A. R., Schmutz, S. M., \& Leeb, T. (2005). Polymorphisms within the canine MLPH gene are associated with dilute coat color in dogs. Bio Med Central Genetics, 6, 34. PMid:15960853.

Roperto, F., Cerundolo, R., Restucci, B., Vincensi, M. R., Caprariis, D., Vico, G. D., \& Maiolino, P. (1995). Colour Dilution Alopecia (CDA) in Ten yorkshire Terriers. Veterinary Dermatology, 6(4), 171-178. http://dx.doi. org/10.1111/j.1365-3164.1995.tb00062.x. 